\title{
Integrated Flexible Ocular Coil for Power and Data Transfer in Retinal Prostheses
}

\author{
W. $\mathrm{Li}^{1}$, D. C. Rodger ${ }^{2,3}$, J. D. Weiland ${ }^{3}$, M. S. Humayun ${ }^{3}$, and Y. C. Tai ${ }^{1,2}$ \\ ${ }^{1}$ Electrical Engineering, Div. of Engineering and Applied Science, California Institute of Technology, Pasadena, CA, USA \\ ${ }^{2}$ Bioengineering, Div. of Engineering and Applied Science, California Institute of Technology, Pasadena, CA, USA \\ ${ }^{3}$ Keck School of Medicine, University of Southern California, Los Angeles, CA, USA
}

\begin{abstract}
A microfabricated and fully-implantable coil for use as a power and data transfer component for retinal prostheses is presented. Compared with traditional hand-made ocular coils, this parylene-based device is thin and flexible with 10 turns of thin-film metal wires and a thickness of less than $10 \mu \mathrm{m}$. In addition, the entire coil structure can be heat-formed on a mold to match the eye's curvature for extraocular implantation. Because it is made using parylene thin-film technology, this coil can be directly integrated with multielectrode arrays and with parylene-based packages incorporating application specific integrated circuits (ASICs) or discrete electrical components such as chip capacitors. This coil thus enables the fabrication and implantation of a fully microfabricated system for retinal prostheses.
\end{abstract}

Keywords - coil, flexible, integration, ocular, parylene, retinal prostheses

\section{INTRODUCTION}

Millions of people worldwide suffer from vision loss due to retinitis pigmentosa (RP) and age-related macular degeneration (AMD) diseases that primarily affect the photoreceptors of the retina. Recently, a retinal prosthesis has been developed for the treatment of age-related blindness. This technology is based on the concept of replacing photoreceptor function with an electronic device [1]. Instead of using a battery, inductive coupling of a pair of coils is a possible mechanism of transferring both power and data wirelessly to the implanted components of the retinal prostheses. In such an implementation, a primary coil resides outside the body, and thus has considerably fewer design constraints in terms of number of turns and power consumption than the implanted components. However, the secondary coil, which will be implanted into human's body, has many constraints such as comfort, very small size, and high coupling efficiency. Currently, only hand-made ocular coils are employed to accomplish the function of this implanted device [2]. These hand-made coils are thick and stiff, and can cause notable mechanical damage in the region of implantation.

To fulfill the mechanical, electrical, and biocompatible requirements of such an implantable coil, we are developing a new parylene-based coil technology incorporating thin-film metal conductive wires. Fig. 1 gives an overall depiction of the device. In this design, at least two layers of metal are needed in order to fabricate a closed circuit with two perimeter contacts. Parylene $\mathrm{C}$ is selected as the encapsulation material because of its desired properties, such as flexibility (Young's modulus $\sim 4 \mathrm{GPa}$ ), chemical inertness, and biocompatibility [3]. In fact, upon intraocular implantation in two rabbits for six months, parylene $\mathrm{C}$ was shown to generate no detectable immune response affecting their retinas [4]. Thin-film micro-electro-mechanical systems (MEMS) technology has several advantages over traditional fabrication approaches for the ocular coil including smaller size, precise dimensional control, and feasibility for system integration.

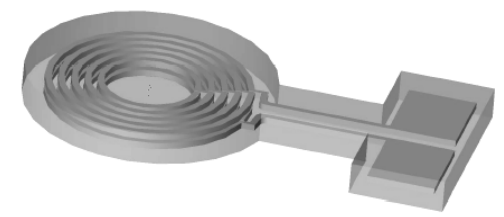

Fig. 1. Illustration of the flexible ocular coil concept.

This coil will be microfabricated alongside multielectrode arrays, packaged application specific integrated circuits (ASICs), and discrete components, such as chip capacitors, to manufacture monolithic flexible retinal system in a single fabrication run [4]. Because of the possibility of fabrication and implantation of such microfabricated coils of many different sizes, this technology is extendable to both the extraocular and intraocular paradigms. Fig. 2 gives a schematic of the implanted retinal system, in which (a) is for the extraocular coil system and (b) depicts the intraocular coil system.

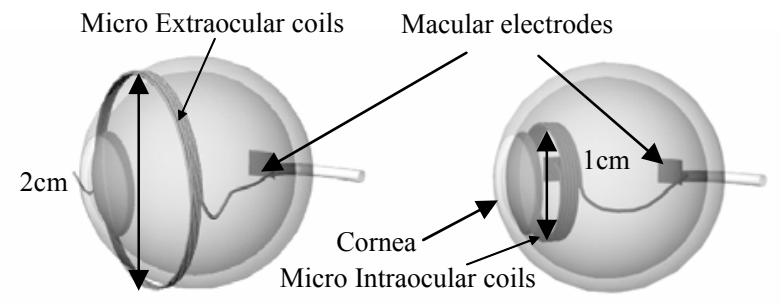

(a)

(b)

Fig. 2. Schematics for microfabricated flexible coils: (a) extraocular coil; (b) intraocular coil.

\section{DEVICE FABRICATION}

The basic technology involved in ocular coil fabrication is the parylene/metal skin technique $[5,6]$. The simplified 
fabrication process is illustrated in Fig. 3. First, photoresist was spun as a sacrificial layer and a parylene layer was deposited on the photoresist. A $220 \mathrm{~nm}$ multi-layer of $\mathrm{Cr} / \mathrm{Au} / \mathrm{Cr}$ was then deposited on the parylene using e-beam evaporation, followed by metal etching process. After that, a $1 \mu \mathrm{m}$ insulating layer of parylene was deposited on the metal, and then was patterned using oxygen plasma etching to open the contact hole that will be used to connect the two layers of metal. The second layer of metal was then deposited using optimized step coverage and patterned using a photoresist mask. Parylene deposition was then performed so that the metals were entirely encapsulated. The parylene was then patterned using oxygen plasma to shape the coil profile. Finally, the whole flexible skin was released from the silicon substrate by dissolving the photoresist sacrificial layer using a standard acetone etch process.

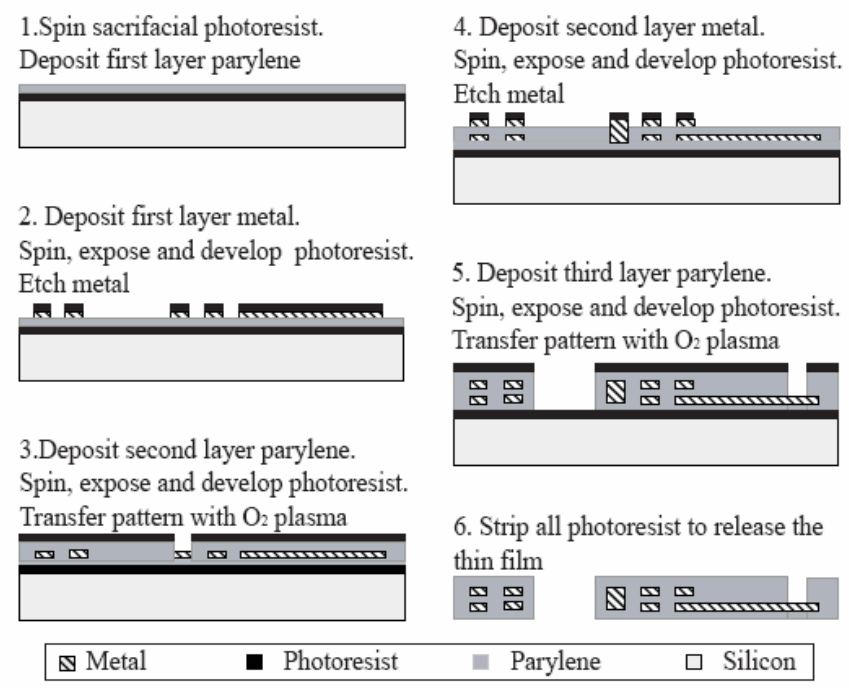

Fig. 3. Metal etch process for ocular coils fabrication.

A fabricated flexible ocular coil is shown in Fig. 4(a), and Fig. 4(b) shows the location of the interconnection between the two layers of metals. This microfabricated coil has 10 turns of metal wires coated with $10-\mu \mathrm{m}$-thick parylene, and is $9.5 \mathrm{~mm}$ in outer diameter. Compared with hand-made coils, this parylene/metal skin is flexible and foldable due to the unique properties of the parylene. Fig. 5 demonstrates the flexibility of this coil.

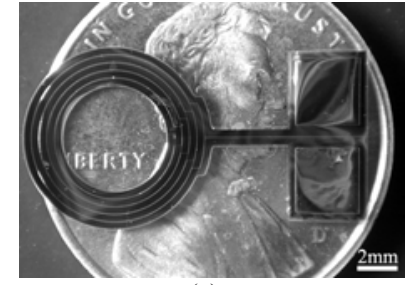

(a)

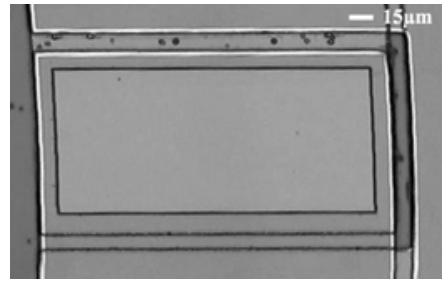

(b)
Fig. 4. (a) Fabricated ocular coil sitting on a penny; (b) Interconnection pad between two layers of metals.

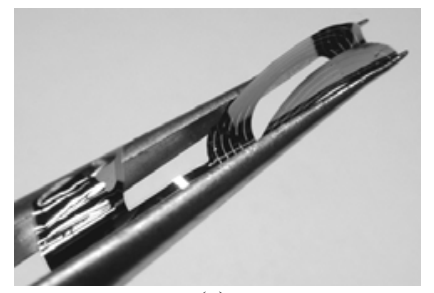

(a)

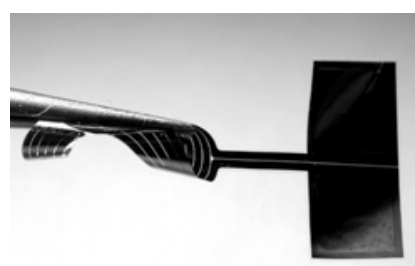

(b)
Fig. 5. Flexibility of the coil: (a) skin folded in half; (b) skin rolled into a tube.

\section{RESULTS AND DISCUSSION}

\section{A. Heat-forming treatment}

For extraocular coil applications, the device will be implanted on the outer surface of the eye. Therefore, the coil needs to be modified into a curvature similar to that of the eye to satisfy biocompatibility and comfort requirements. Instead of using prohibitively-difficult 3D fabrication techniques, the parylene/metal skin can be directly heat-formed on an existing metal mold to match the eye's curvature. Different coil curvatures can be achieved by varying the size of molds to match the actual dimensions of the human's eye. To prevent the parylene from being oxidized in air at the high temperatures necessary for heat-forming, this treatment is performed in a vacuum oven with $\mathrm{N}_{2}$ backfill. The heating temperature should be lower than the melting temperature of parylene C. Fig. 6 shows a coil after heat-forming at $190{ }^{\circ} \mathrm{C}$ for 15 hours.

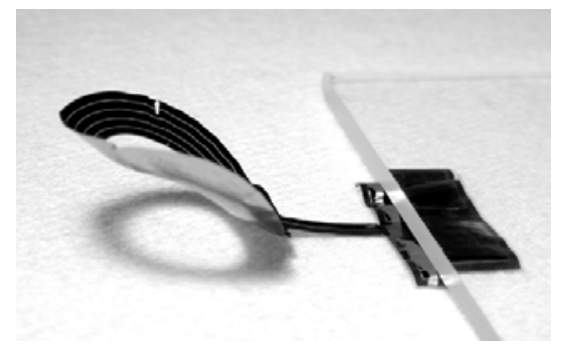

Fig. 6. Coil heat-formed at $190^{\circ} \mathrm{C}$.

\section{B. Electrical characteristics}

The series resistances and self-inductances of the coils were measured using an HP 4145B and HP 4192A respectively. As expected from the design, the average DC resistance of this ocular coil is $81.4 \Omega$. The measured I-V curves for a fabricated coil before and after heat-forming at $190{ }^{\circ} \mathrm{C}$ are shown in Fig. 7. It can be seen that the series resistance can be reduced significantly by metal annealing at the high temperature. Fig. 8 shows the self-inductance at different frequencies. At the target frequency of $1 \mathrm{MHz}$ for the current retinal prostheses system, the self-inductance is approximately $0.5 \mu \mathrm{H}$. 


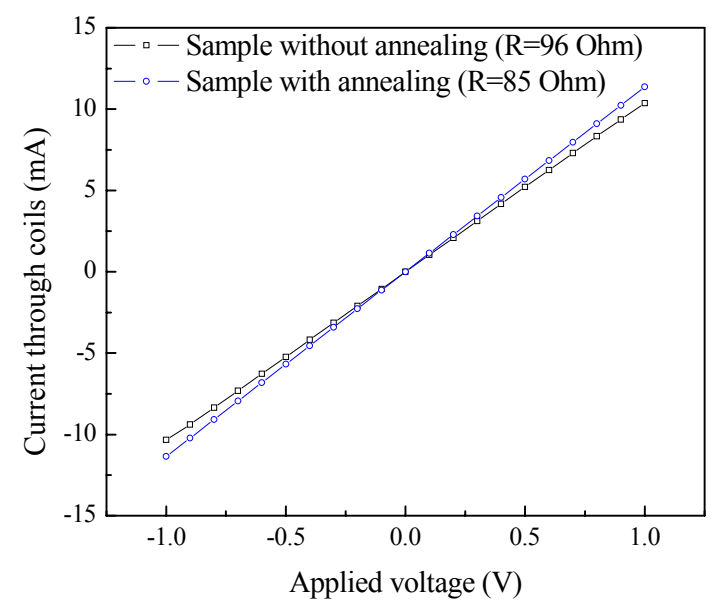

Fig. 7. Measured I-V curves before and after heat-forming.

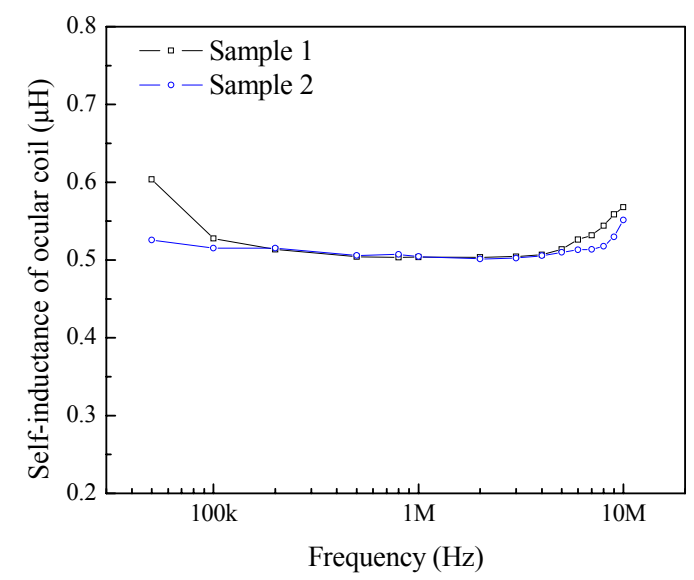

Fig. 8. Self-inductances of ocular coil vs. frequency.

A simplified inductive link has been built to verify the coupling effect, as illustrated in Fig. 9(a). The primary coil is hand-made, and has a self-inductance of $23.1 \mu \mathrm{H}$, while the secondary coil is microfabricated using the parylene/metal thin film process described here. The primary coil is driven by a standard function generator. Both the primary and secondary coils are subject to parallel resonance. The output voltage and the corresponding output power across a $1 \mathrm{k} \Omega$ load resistor, as a function of the coil separation distance, is plotted in Fig. 9(b). In this test, the input voltage of the primary coil was a sinusoidal signal with peak amplitude of $10 \mathrm{~V}$, and both circuits were tuned to the resonance frequency of $1 \mathrm{MHz}$.

This preliminary experiment has demonstrated that the consistent power can be transmitted using our flexible ocular coils. The small output power is mainly due to the low inductance of secondary coil. Moreover, the large series resistance results in large power consumption in the secondary coil, which can cause the undesired temperature increases and potential thermal damage upon implantation. To improve the performance of future version of the flexible ocular coil, several possible modifications will be made. Work is currently focused on enhancing the inductance, and reducing the series resistance of the coils. Electroplating technology can be employed for metal deposition to achieve lower series resistances in the coils and higher power transfer efficiency. To improve the inductance of the coils, a stack structure comprising more than two layers of metal can be made by alternating the parylene deposition, interconnection hole fabrication, and metal evaporation process steps.

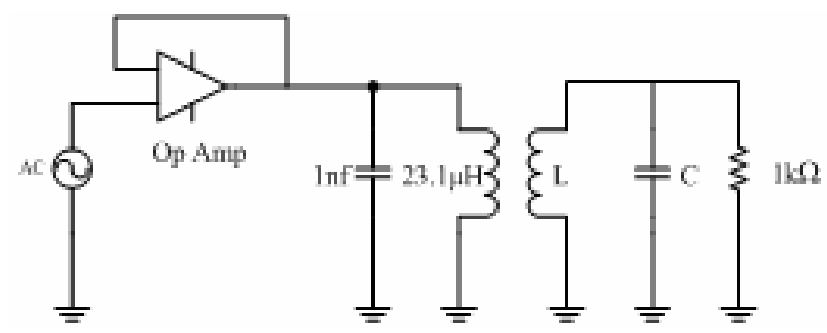

(a)

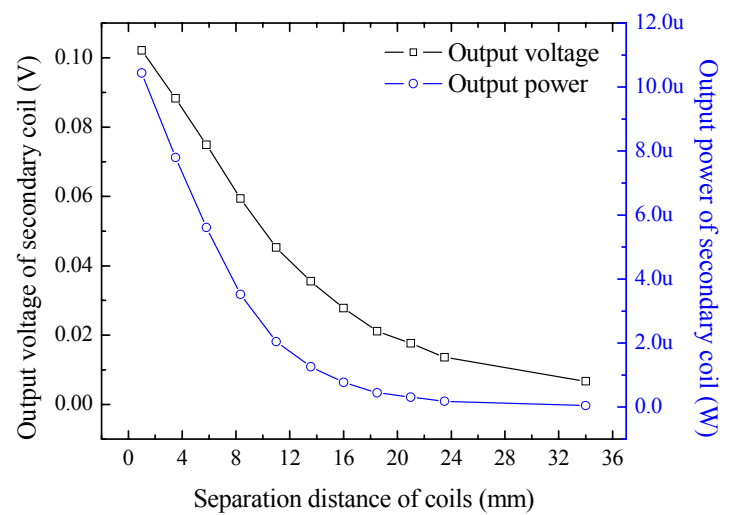

(b)

Fig. 9. Inductive coupling: (a) Schematic for transmission circuit; (b) Output voltage and output power vs. axial coil displacement.

\section{CONCLUSION}

A novel ocular coil for retinal prostheses has been fabricated using the parylene/metal skin microfabrication technique. Because of the unique properties of parylene, this device is flexible and foldable so as to enable implantation into the eye through a small surgical incision. Furthermore, the parylene/metal skin can be directly heat-formed to match the curvatures in the region of implantation after fabrication. Preliminary test results have demonstrated that power can be consistently transmitted using this device. Although specifically tailored to the needs of retinal prostheses, because parylene thin-film technology is used for coil fabrication, and because this coil can be integrated with MEMS devices, discrete electrical components, and ASICs, it enables a new range of true system solutions for both biomedical and non-biomedical applications. 


\section{ACKNOWLEDGMENT}

This work was supported in part by the Engineering Research Center Program of the National Science Foundation under Award Number EEC-0310723 and by a fellowship from the Whitaker Foundation (D.R.). The authors would also like to thank Mr. Trevor Roper and other members of the Caltech Micromachining Laboratory for assistance with fabrication.

\section{REFERENCES}

[1] M. S. Humayun, "Intraocular retinal prosthesis," Tr. Am Ophth Soc, vol. 99, pp. 271-300, 2001.

[2] T. Stieglitz, W. Haberer, C. Lau, and M. Goertz, "Development of an inductively coupled epiretinal vision prosthesis," in Proc. 26th Int. Conf. IEEE Eng. in Med. and Biol. Soc., San Francisco, California, USA, pp.4178-4181, 2004.
[3] L. Wolgemuth, "Assessing the performance and suitability of parylene coating," Medical Device \& Diagnostic Industry, vol. 22, pp. 42-49, 2000.

[4] D. C. Rodger, J. D. Weiland, M. S. Humayun, and Y. C. Tai, "Scalable flexible chip-level parylene package for high lead count retinal prostheses," in Proc. 13th Int. Conf. on Solid-State Sensors, Actuators, and Microsystems, Seoul, Korea, pp. 1973-1976, 2005.

[5] M. Liger, N. Pornsinsirirak, Y. C. Tai, S. Ho and C. M. Ho, "Large-area electrostatically-valved skins for adaptive flow control on ornithopter wings," Technical Digest, Solid State Sensor and Actuator Workshop, Hilton Head Island, South Carolina, USA, pp. 247-250, 2002.

[6] Y. C. Tai, F. Jiang, Y. Xu, M. Liger, S. Ho and C. M. Ho, "Flexible MEMS skins: technologies and applications," Proceedings, Pacific Rim MEMS Workshop, Xiamen, China, 2002. 